\title{
Wall Temperature Effects on the Reynold Stress of Flat-Plate Turbulent Boundary Layer: a Numerical Investigation
}

\author{
Xin $\mathrm{Li}^{1,2}$, Changping $\mathrm{Yu}^{1,2}$ and Xinliang $\mathrm{Li}^{1,2 *}$ \\ ${ }^{1}$ LHD, Institute of Mechanics, Chinese Academy of Sciences, Beijing 100190, China \\ 2 School of Engineering Science, University of Chinese Academy of Sciences, \\ Beijing 100049, China
}

Received 20 August 2018; Accepted (in revised version) 6 December 2018

\begin{abstract}
In this paper, direct numerical simulation (DNS) of spatially evolving flatplate hypersonic turbulent boundary layer with free-stream Mach number 8 is used to study the effects of wall temperature on compressibility and Reynolds stress contribution. The DNS contains two cases with different wall temperatures, $T_{w} / T_{\infty}=10.03$ and 1.9. The results show that lower wall temperature enhances both the compressibility and injection-and-sweeping motions near the wall.
\end{abstract}

AMS subject classifications: 76K05

Key words: Hypersonic, direct numerical simulation, wall temperature, Reynold stress.

\section{Introduction}

Compressible turbulent boundary-layer flows are typical flows in supersonic and hypersonic vehicles. In hypersonic flows, wall temperature increases rapidly by friction dissipation and wall-cooling techniques are used to protect the aircrafts. As known by represent researches [1-4], surface temperature significantly affects the statistics and coherent structures of hypersonic turbulent boundary layer. The validity of Morkovin's hypothesis was checked at moderate free-stream Mach numbers (Mach $\leq 5)[5,6]$, but it is not studied with cooling situations [4]. Constrained by the very short measurement time and low resolution measuring means, only some limited experimental studies [7-9] at high Mach numbers 7.2 and 11, respectively, focused on the applicability of Morkovin's hypothesis for compressible turbulent boundary layers. Experimental studies for hypersonic transitions are performed by using hypersonic quiet wind tunnel, and the relationship between the local high heat flux and Mack 2nd waves are revealed $[10,11]$.

*Corresponding author.

Emails: lixin@imech.ac.cn (X. Li), cpyu@imech.ac.cn (C. P. Yu), lixl@imech.ac.cn (X. L. Li) 
Direct numerical simulation (DNS) is a powerful tool to study the turbulence mechanism. However, due to the huge computing cost, most DNS studies focused on low and middle Mach number conditions [4,5,12-14]. For example, Pirozzoli [5] conducted systematic analyses of mean and statistical behavior of turbulent boundary layer at Mach 2.25. Duan [4] studied five different wall-to-recovery temperature ratios $T_{w} / T_{r}$ from 0.18 to 1 and obtained productive results. Recently, some researches focused on hypersonic flows $[15,16]$. They performed DNS of the effects of cold-wall conditions on vorticity and pressure fluctuations, respectively. From the results above, DNS study of spatially evolving hypersonic turbulent boundary layer on wall cooling is limited. It is very necessary to carry out DNS study in this field.

Compressibility effects are important in hypersonic flows, and deeper researching on it is also very helpful for turbulence modelling. In this study, DNS of Mach 8 flatplate boundary layer with two wall temperatures conditions $T_{w} / T_{\infty}=10.03$ and 1.9 are performed to study the effects of wall temperature on the compressibility and Reynold stress in turbulent boundary layer.

\section{Numerical method and computational condition}

In the DNS, a high-order finite difference code developed by the authors, OpenCFD-SC, is used. Spatial discretizations of convection terms and viscous terms use WENO-JS7 scheme [17] and the $8^{\text {th }}$-order central difference, respectively. More details can be found in $[2,3]$.

Table 1: Computational conditions and grid parameters for the DNS.

\begin{tabular}{||cccccc||}
\hline Case & $T_{w} / T_{\infty}$ & $M a_{\delta}$ & $L_{x} \times L_{y} \times L_{z}$ & $N_{x} \times N_{y} \times N_{z}$ & $\Delta_{x}^{+} \times \Delta_{y}^{+} \times \Delta_{z}^{+}$ \\
\hline TH & 10.03 & 6.92 & $37 \times 0.7 \times 0.3$ & $12460 \times 100 \times 320$ & $12.2 \times 0.96 \times 4.6$ \\
TL & 1.9 & 7.29 & $11 \times 0.7 \times 0.18$ & $8950 \times 90 \times 640$ & $11.2 \times 1.0 \times 4.5$ \\
\hline
\end{tabular}

The DNS parameters are shown in Table 1, where $T H$ denotes the high temperature case, TL denotes the low wall temperature case, $\infty$ represents free stream, and $\delta$ is boundary layer thickness that is defined as the $y$-direction value of 0.99 freestream velocity. For the high and low temperature cases, the normalized boundary layer thicknesses are set to $\delta=0.0246$, and 0.0122 by inch, respectively, with both cases being in fully developed turbulent states.

\section{Results and discussions}

Figs. 1(a) and (b) show the skin friction coefficients $\left(C_{f}\right)$ for both two wall temperature cases, which shows that the boundary layer flows transition to the fully developed states. These results get well agreement with effective theoretical results by White [18], which 